\title{
ON THE PROPERTIES OF THE EXCHANGE GRAPH OF A CLUSTER ALGEBRA
}

\author{
Michael Gekhtman, Michael Shapiro, and Alek Vainshtein
}

Abstract. We prove a conjecture about the vertices and edges of the exchange graph of a cluster algebra $\mathcal{A}$ in two cases: when $\mathcal{A}$ is of geometric type and when $\mathcal{A}$ is arbitrary and its exchange matrix is nondegenerate. In the second case we also prove that the exchange graph does not depend on the coefficients of $\mathcal{A}$. Both conjectures were formulated recently by Fomin and Zelevinsky.

\section{Main definitions and results}

A cluster algebra is an axiomatically defined commutative ring equipped with a distinguished set of generators (cluster variables). These generators are subdivided into overlapping subsets (clusters) of the same cardinality that are connected via sequences of birational transformations of a particular kind, called cluster transformations. Transfomations of this kind can be observed in many areas of mathematics (Plücker relations, Somos sequences and Hirota equations, to name just a few examples).

Cluster algebras were initially introduced in [FZ1] to study total positivity and (dual) canonical bases in semisimple algebraic groups. The rapid development of the cluster algebra theory revealed relations between cluster algebras and Grassmannians, quiver representations, generalized associahedra, Teichmüller theory, Poisson geometry and many other branches of mathematics, see [Ze] and references therein. In the present paper we prove some conjectures on the general structure of cluster algebras formulated by Fomin and Zelevinsky in [FZ3].

To state our results, we recall the definition of a cluster algebra; for details see [FZ1, FZ4].

Let $\mathcal{P}$ be a semifield, that is, a torsion-free multiplicative abelian group endowed with an additional operation $\oplus$, which is commutative, associative and distributive with respect to the multiplication. As an ambient field we take the field $\mathcal{F}$ of rational functions in $n$ independent variables with coefficients in the field of fractions of the integer group ring $\mathbb{Z P}$. A square matrix $B$ is called skew-symmetrizable if $D B$ is skew-symmetric for a non-degenerate non-negative diagonal matrix $D$. A seed is a triple $\Sigma=(\mathbf{x}, \mathbf{y}, B)$, where $\mathbf{x}=\left(x_{1}, \ldots, x_{n}\right)$ is a transcendence basis of $\mathcal{F}$ over the field of fractions of $\mathbb{Z} \mathcal{P}, \mathbf{y}=\left(y_{1}, \ldots, y_{n}\right)$ is an $n$-tuple of elements of $\mathcal{P}$ and $B$ is a

Received by the editors April 1, 2007.

1991 Mathematics Subject Classification. 22E46.

Key words and phrases. Cluster algebra, exchange graph, compatible 2-form.

The authors were supported in part by BSF Grant \#2002375.

The first author was supported in part by NSF Grant \#0400484.

The second author was supported in part by PHY Grant \#0555346 and DMS Grant \#0401178. 
skew-symmetrizable integer $n \times n$ matrix. The components $\mathbf{x}, \mathbf{y}$ and $B$ of the seed are called the cluster, the coefficient tuple and the exchange matrix, respectively; the entries of $\mathbf{x}$ are called cluster variables.

A seed mutation in direction $k \in[1, n]$ takes $\Sigma$ to an adjacent seed $\Sigma^{\prime}=\left(\mathbf{x}^{\prime}, \mathbf{y}^{\prime}, B^{\prime}\right)$ whose components are defined as follows. The adjacent cluster $\mathbf{x}^{\prime}$ is given by $\mathbf{x}^{\prime}=$ $\left(\mathbf{x} \backslash\left\{x_{k}\right\}\right) \cup\left\{x_{k}^{\prime}\right\}$, where the new cluster variable $x_{k}^{\prime}$ is defined by the exchange relation

$$
x_{k} x_{k}^{\prime}=\frac{y_{k}}{y_{k} \oplus 1} \prod_{b_{k i}>0} x_{i}^{b_{k i}}+\frac{1}{y_{k} \oplus 1} \prod_{b_{k i}<0} x_{i}^{-b_{k i}}
$$

where, as usual, the product over the empty set is assumed to be equal to 1 . The adjacent coefficient tuple $\mathbf{y}^{\prime}$ is given by

$$
y_{j}^{\prime}= \begin{cases}y_{k}^{-1} & \text { if } j=k, \\ y_{j} y_{k}^{b_{j k}}\left(y_{k} \oplus 1\right)^{-b_{j k}} & \text { if } j \neq k \text { and } b_{j k}>0, \\ y_{j}\left(y_{k} \oplus 1\right)^{-b_{j k}} & \text { if } j \neq k \text { and } b_{j k} \leq 0 .\end{cases}
$$

Finally, the adjacent exchange matrix $B^{\prime}$ is given by

$$
b_{i j}^{\prime}= \begin{cases}-b_{i j}, & \text { if } i=k \text { or } j=k \\ b_{i j}+\frac{\left|b_{i k}\right| b_{k j}+b_{i k}\left|b_{k j}\right|}{2}, & \text { otherwise. }\end{cases}
$$

Remark. Note that our encoding of exponents in relations 1 and 2 by the entries of $B$ differs from the one used in [FZ1]-[FZ4] by transposition of subscripts. In other words, our exchange matrix is the transpose of the one used in [FZ1]-[FZ4].

Two seeds are called mutation equivalent if they can be obtained one from another by a sequence of seed mutations. The cluster algebra $\mathcal{A}=\mathcal{A}(\Sigma)$ associated with $\Sigma$ is the $\mathbb{Z} \mathcal{P}$-subalgebra of $\mathcal{F}$ generated by all cluster variables in all seeds mutation equivalent to $\Sigma ; n$ is said to be the rank of $\mathcal{A}(\Sigma)$. by

We say that $\mathcal{A}$ is of geometric type if $\mathcal{P}$ is a tropical semifield, that is, if $\oplus$ is defined

$$
\prod_{i=1}^{m} g_{i}^{a_{i}} \oplus \prod_{i=1}^{m} g_{i}^{b_{i}}=\prod_{i=1}^{m} g_{i}^{\min \left(a_{i}, b_{i}\right)} .
$$

For a cluster algebra of geometric type, each entry of the coefficient tuple $\mathbf{y}$ can be represented as

$$
y_{i}=\prod_{i=1}^{m} g_{j}^{a_{i j}}
$$

for some integer $a_{i j}$. Re-denote the generators $g_{1}, \ldots, g_{m}$ by $x_{n+1}, \ldots, x_{n+m}$ and call them stable variables; together with the cluster variables, they form an extended cluster. Define an extended exchange matrix $\widetilde{B}$ as an $n \times(n+m)$ matrix whose $(i, j)$ th entry equals $b_{i j}$ for $i \in[1, n]$ and $a_{i-n, j}$ for $i \in[n+1, n+m]$. For an arbitrary matrix $A$, denote by $A[k ; l]$ the submatrix of $A$ occupying the first $k$ rows and the first $l$ columns; with this notation, the relation between $B$ and $\widetilde{B}$ is given by $B=\widetilde{B}[n ; n]$.

Transformation rules (1) can be rewritten for cluster algebras of geometric type as

$$
x_{k} x_{k}^{\prime}=\prod_{\substack{1 \leq i \leq n+m \\ b_{k i}>0}} x_{i}^{b_{k i}}+\prod_{\substack{1 \leq i \leq n+m \\ b_{k i}<0}} x_{i}^{-b_{k i}} .
$$


Transformation rules (2) imply that mutations of the extended exchange matrix are governed by the same matrix mutation rules (3). The corresponding cluster algebra of geometric type is denoted $\mathcal{A}(\widetilde{B})$.

A convenient tool in dealing with cluster algebras is the $n$-regular tree $\mathbb{T}_{n}$. Its vertices correspond to seeds, and two vertices are connected by an edge labeled by $k$ if and only if the corresponding seeds are adjacent in direction $k$. The edges of $\mathbb{T}_{n}$ are thus labeled by the numbers $1, \ldots, n$ so that the $n$ edges emanating from each vertex receive different labels. Two seeds differing from each other by an arbitrary permutation of $\mathbf{x}$ and the corresponding permutation of $\mathbf{y}$ and of the rows and columns of $\widetilde{B}$ are called equivalent. The exchange graph of a cluster algebra is defined as the quotient of the tree $\mathbb{T}_{n}$ modulo this equivalence relation.

The following conjectures about the exchange graph of a cluster algrebra were formulated in [FZ3].

Conjecture 1. The exchange graph of a cluster algebra depends only on the initial exchange matrix $B$.

Conjecture 2. Every seed is uniquely defined by its cluster; thus, the vertices of the exchange graph can be identified with the clusters, up to a permutation of cluster variables.

Conjecture 3. Two clusters are adjacent in the exchange graph if and only if they have exactly $n-1$ common cluster variables.

All three conjectures were proved for cluster algebras of finite type in [FZ2], and for cluster algebras associated with triangulations of two-dimensional surfaces in [FST]. Besides, in [BMRT] Conjectures 2 and 3 were proved for cluster algebras of geometric type with no stable variables with a skew-symmetric exchange matrix satisfying an additional acyclicity condition.

In this note we prove the following three results. Denote by $\mathfrak{A}(B)$ the family of all cluster algebras obtained by fixing the initial exchange matrix $B$ and varying the semifield $\mathcal{P}$, the choice of the initial coefficient $n$-tuple $y$, and the choice of the initial cluster $x$ (i.e., the choice of a transcendence basis of $\mathcal{F}$ over the field of fractions of $\mathbb{Z} \mathcal{P})$.

Theorem 4. Let a cluster algebra $\mathcal{A} \in \mathfrak{A}(B)$ satisfy one of the following two conditions:

(i) $\mathcal{A}$ is of geometric type;

(ii) $\mathcal{A}$ is arbitrary and $B$ is nondegenerate.

Then every seed in $\mathcal{A}$ is uniquely defined by its cluster (in other words, Conjecture 2 holds true for $\mathcal{A}$ ).

Theorem 5. Suppose that every seed in a cluster algebra $\mathcal{A}$ is uniquely defined by its cluster. Then two clusters are adjacent in the exchange graph of $\mathcal{A}$ if and only if they have exactly $n-1$ common variables. In particular, Conjecture 3 holds true if $\mathcal{A}$ satisfies one of the conditions (i), (ii) of Theorem 4.

Remark. Note that we do not claim that the whole cluster complex is determined by set-theoretic combinatorics of subsets (clusters) of the ground set of all cluster variables, although we strongly believe that it is true. Theorem 5 states only that 
the exchange graph of a cluster algebra is determined by this combinatorics. In other words, we can restore which maximal simplices are adjacent by a codimension one face.

Theorem 6. Let $B$ be nondegenerate, then the exchange graphs of all cluster algebras in $\mathfrak{A}(B)$ coincide (in other words, Conjecture 1 holds true).

We prove Theorems 4-6 using the concept of a 2-form compatible with the cluster algebra structure [GSV1, GSV2]. Under certain nondegeneracy conditions, this approach has already proved useful in realizing cluster algebras as coordinate rings of rational Poisson manifolds.

\section{Proof of Theorem 4}

In what follows we write $x_{i ; \Sigma}, y_{i ; \Sigma}$ and $b_{i j ; \Sigma}$ to indicate that the corresponding cluster variables, coefficients and entries of the exchange matrix are related to a seed $\Sigma$.

Observe that Conjecture 2 is equivalent to the following one: suppose that the seeds $\Sigma_{1}$ and $\Sigma_{2}$ satisfy relations

$$
x_{i ; \Sigma_{2}}=x_{\sigma(i) ; \Sigma_{1}}
$$

for some $\sigma \in S_{n}$ and any $i \in[1, n]$, then

$$
y_{i ; \Sigma_{2}}=y_{\sigma(i) ; \Sigma_{1}}
$$

for $i \in[1, n]$ and

$$
b_{i j ; \Sigma_{2}}=b_{\sigma(i) \sigma(j) ; \Sigma_{1}}
$$

for any $i, j \in[1, n]$.

First, consider case (i) in Theorem 4. Following [GSV2], we say that a closed rational differential 2-form $\omega$ on the $(n+m)$-dimensional affine space is compatible with the cluster algebra $\mathcal{A}(\widetilde{B})$ if for any extended cluster $\widetilde{\mathbf{x}}=\left(x_{1}, \ldots, x_{n+m}\right)$ one has

$$
\omega=\sum_{i, j=1}^{n+m} \omega_{i j} \frac{d x_{i}}{x_{i}} \wedge \frac{d x_{j}}{x_{j}},
$$

where $\omega_{i j}$ are constants (recall that $x_{i}$ 's are rational functions in the initial cluster variables). The matrix $\Omega=\left(\omega_{i j}\right)$ is called the coefficient matrix of $\omega$ (with respect to $\widetilde{\mathbf{x}})$; evidently, $\Omega$ is skew-symmetric.

A square matrix $A$ is decomposable if there exists a permutation matrix $P$ such that $P A P^{T}$ is a block-diagonal matrix, and indecomposable otherwise; $\rho(A)$ is defined as the maximal number of diagonal blocks in $P A P^{T}$. The partition into blocks defines an obvious equivalence relation $\sim$ on the rows (or columns) of $A$.

The following result is a generalization of Theorem 2.1 of [GSV2].

Theorem 7. Assume that $\widetilde{B}$ is D-skew-symmetrizable and does not have zero rows. Then all rational closed 2-forms compatible with $\mathcal{A}(\widetilde{B})$ form a vector space of dimension $\rho(B)+\left(\begin{array}{c}m \\ 2\end{array}\right)$, where $B=\widetilde{B}[n ; n]$. Moreover, the coefficient matrices of these 2-forms with respect to $\widetilde{\mathbf{x}}$ are characterized by the equation $\Omega[n ; n+m]=\Lambda D \widetilde{B}$, where $\Lambda=\operatorname{diag}\left(\lambda_{1}, \ldots, \lambda_{n}\right)$ with $\lambda_{i}=\lambda_{j}$ whenever $i \sim j$. In particular, if $B$ is indecomposable, then $\Omega[n ; n+m]=\lambda D \widetilde{B}$. 
Proof. Indeed, let $\omega$ be a 2 -form compatible with $\mathcal{A}(\widetilde{B})$. Then

$$
\omega=\sum_{j, k=1}^{n+m} \omega_{j k} \frac{d x_{j}}{x_{j}} \wedge \frac{d x_{k}}{x_{k}}=\sum_{j, k=1}^{n+m} \omega_{j k}^{\prime} \frac{d x_{j}^{\prime}}{x_{j}^{\prime}} \wedge \frac{d x_{k}^{\prime}}{x_{k}^{\prime}},
$$

where $x_{j}^{\prime}$ is given by (5) and $\omega_{j k}^{\prime}$ are the coefficients of $\omega$ with respect to $\widetilde{\mathbf{x}}^{\prime}$. Recall that the only variable in the extended cluster $\widetilde{\mathbf{x}}^{\prime}$ different from the corresponding variable in $\widetilde{\mathbf{x}}$ is $x_{i}$, and

$$
\frac{d x_{i}^{\prime}}{x_{i}^{\prime}}=-\frac{d x_{i}}{x_{i}}+\sum_{b_{i k}>0} \frac{b_{i k}}{1+\prod_{k=1}^{n+m} x_{k}^{-b_{i k}}} \frac{d x_{k}}{x_{k}}-\sum_{b_{i k}<0} \frac{b_{i k}}{1+\prod_{k=1}^{n+m} x_{k}^{b_{i k}}} \frac{d x_{k}}{x_{k}} .
$$

Thus, for any $j \in[1, n+m]$ we immediately get

$$
\omega_{i j}^{\prime}=-\omega_{i j}
$$

Next, consider any pair $j, k \neq i$ such that both $b_{i j}$ and $b_{i k}$ are nonnegative, and at least one of the two is positive. Then

$$
\omega_{j k}=\omega_{j k}^{\prime}+\frac{\omega_{i k}^{\prime} b_{i j}+\omega_{j i}^{\prime} b_{i k}}{1+\prod_{k=1}^{n+m} x_{k}^{-b_{i k}}} .
$$

This equality can only hold if $\omega_{i k}^{\prime} b_{i j}+\omega_{j i}^{\prime} b_{i k}=0$, which by (9) is equivalent to $\omega_{i j} b_{i k}=\omega_{i k} b_{i j}$. If both $b_{i j}$ and $b_{i k}$ are positive, this gives

$$
\frac{\omega_{i j}}{b_{i j}}=\frac{\omega_{i k}}{b_{i k}}=\mu_{i} .
$$

Otherwise, if, say, $b_{i j}=0$, one gets $\omega_{i j}=0$. Besides, in any case $\omega_{j k}^{\prime}=\omega_{j k}$.

Similarly, if both $b_{i j}$ and $b_{i k}$ are nonpositive, and at least one of the two is negative, then

$$
\omega_{j k}=\omega_{j k}^{\prime}-\frac{\omega_{i k}^{\prime}+\omega_{j i}^{\prime} b_{i k}}{1+\prod_{k=1}^{n+m} x_{k}^{b_{i k}}}
$$

and hence the same relations as above hold true.

Finally, let $b_{i j} \cdot b_{i k}<0$, say, $b_{i j}>0$ and $b_{i k}<0$; then

$$
\omega_{j k}=\omega_{j k}^{\prime}+\frac{\omega_{i k}^{\prime} b_{i j} \prod_{k=1}^{n+m} x_{k}^{b_{i k}}-\omega_{j i}^{\prime} b_{i k}}{1+\prod_{k=1}^{n+m} x_{k}^{b_{i k}}},
$$

which again leads to (10); the only difference is that in this case $\omega_{j k}^{\prime}=\omega_{j k}+\omega_{i k} b_{i j}$.

We have thus obtained that $\Omega[n ; n+m]=\operatorname{diag}\left(\mu_{1}, \ldots, \mu_{n}\right) \widetilde{B}$. Recall that $\Omega$ is skew-symmetric; however, any skew-symmetrizer for $\widetilde{B}$ can be written as $\Lambda D$, where $\Lambda=\operatorname{diag}\left(\lambda_{1}, \ldots, \lambda_{n}\right)$ and $\lambda_{i}=\lambda_{j}$ whenever $i \sim j$, which completes the proof.

Assume now that the initial extended matrix $\widetilde{B}$ does not have zero rows. Then by Theorem 7 we can pick a rational closed 2-form $\omega$ compatible with $\mathcal{A}$ so that for any seed $\Sigma$ holds

$$
B_{\Sigma}=D^{-1} \Omega_{\Sigma}[n ; n+m],
$$

where $D$ is a skew-symmetrizer of $B$ and $\Omega_{\Sigma}$ stands for the coefficient matrix of $\Omega$ with respect to $\widetilde{\mathbf{x}}_{\Sigma}$. Since (6) implies $\omega_{i j ; \Sigma_{2}}=\omega_{\sigma(i) \sigma(j) ; \Sigma_{1}}$, we get

$$
b_{\sigma(i) \sigma(j) ; \Sigma_{1}}=d_{\sigma(i)}^{-1} \omega_{\sigma(i) \sigma(j) ; \Sigma_{1}}=d_{\sigma(i)}^{-1} \omega_{i j ; \Sigma_{2}}=d_{i} d_{\sigma(i)}^{-1} b_{i j ; \Sigma_{2}} .
$$


Let us show that $d_{i}=d_{\sigma(i)}$. Indeed, relation (12) implies that $\sigma D$ is a skewsymmetrizer for $B$ along with $D$. If $B$ is indecomposable then any two skew-symmetrizers coincide up to the multiplication by a positive constant, hence $d_{i}=\lambda d_{\sigma(i)}$ for some $\lambda>0$. Iterating this equality we get $\lambda^{k}=1$ for some integer $k>1$, and hence $\lambda=1$. If $B$ is decomposable then the same holds for any matrix mutation equivalent to $B$ with the same partition into blocks, and hence $\sigma$ cannot permute cluster variables from different blocks. Inside each block the previous reasoning remains valid. We thus proved that (8) holds true for any $i, j \in[1, n]$. Similarly, taking into account (11) and $d_{i}=d_{\sigma(i)}$ we get $b_{i, n+j ; \Sigma_{2}}=b_{\sigma(i), n+j ; \Sigma_{1}}$ for any $i, j \in[1, n]$. It remains to recall that for cluster algebras of geometric type the $n$-tuple $\mathbf{y}_{\Sigma}$ is completely defined by the matrix $B_{\Sigma}$ via (4).

Assume now that $\widetilde{B}$ has $k$ zero rows. In this case $\mathcal{A}$ is a direct product of $k$ copies of the cluster algebra of rank 1 and a cluster algebra defined by a $(n-k) \times(n+m)$ submatrix of $\widetilde{B}$ with no zero rows, for which the above reasoning applies.

Consider now case (ii). Define the coefficient-free cluster algebra $\mathcal{A}_{\mathrm{cf}}(B) \in \mathfrak{A}(B)$ as the cluster algebra over the one-element semifield $\{1\}$ obtained by mutations of the seed $(\mathbf{x}, 1, B)$. Clearly, the map that takes all elements of $\mathcal{P}$ to 1 commutes with the exchange relation (1). Therefore, (6) for an algebra $\mathcal{A} \in \mathfrak{A}(B)$ implies the same relation for $\mathcal{A}_{\mathrm{cf}}(B)$. Since the latter is of geometric type, the result of case (i) applies, and hence relation (8) holds true.

Following [FZ4], for an arbitrary cluster algebra $\mathcal{A} \in \mathfrak{A}(B)$ introduce the $n$-tuple $\widehat{\mathbf{y}}_{\Sigma}=\left(\widehat{y}_{1 ; \Sigma}, \ldots, \widehat{y}_{n ; \Sigma}\right)$ by

$$
\widehat{y}_{j ; \Sigma}=y_{j ; \Sigma} \prod_{k=1}^{n} x_{k ; \Sigma}^{b_{j k \Sigma}} .
$$

For cluster algebras of geometric type, in particular, for the coefficient-free algebra $\mathcal{A}_{\mathrm{cf}}(B)$, this $n$-tuple coincides with $\tau$-coordinates introduced in [GSV1]. Further, define $Y_{i ; \Sigma}\left(y_{1}, \ldots, y_{n}\right)$ as a rational subtraction-free expression for the coefficient $y_{i ; \Sigma}$ via the initial coefficients $y_{1}, \ldots, y_{n}$. The evaluation of $Y_{i ; \Sigma}$ over $\mathcal{P}$ denoted by $\left.Y_{i ; \Sigma}\right|_{\mathcal{P}}$ gives the value of $y_{i ; \Sigma}$ in the cluster algerba over $\mathcal{P}$; this operation is well-defined since any subtraction-free identity in the field of rational functions remains valid in any semifield (see [BFZ], Lemma 2.1.6).

By Proposition 3.9 in [FZ4],

$$
\widehat{y}_{j ; \Sigma}=\left.Y_{j ; \Sigma}\right|_{\mathcal{F}}\left(\widehat{y}_{1}, \ldots, \widehat{y}_{n}\right) .
$$

Therefore, (6) and (8) for $\mathcal{A}_{\mathrm{cf}}(B)$ imply $Y_{i ; \Sigma_{2}}\left(\tau_{1}, \ldots, \tau_{n}\right)=Y_{\sigma(i) ; \Sigma_{1}}\left(\tau_{1}, \ldots, \tau_{n}\right)$.

Since $B$ is nondegenerate, Lemma 1.1 in [GSV1] implies that the transformation $\mathbf{x} \mapsto \tau$ is a bijection, and hence $Y_{i ; \Sigma_{2}}\left(z_{1}, \ldots, z_{n}\right)=Y_{\sigma(i) ; \Sigma_{1}}\left(z_{1}, \ldots, z_{n}\right)$ for any set of variables $z_{1}, \ldots, z_{n}$. Therefore, (7) holds for any cluster algebra $\mathcal{A} \in \mathfrak{A}(B)$.

Remark. An similar approach based on utilizing the bijection $\mathbf{x} \mapsto \tau$ is used in the proof of Proposition 2.7 in [FoG].

\section{Proof of Theorem 5}

Denote by $x_{1}, \ldots, x_{n-1}$ the common variables in the two clusters and by $\dot{x}$ and $\ddot{x}$ the remaining variables; the clusters themselves will be denoted $\dot{\mathbf{x}}$ and $\ddot{\mathbf{x}}$, respectively. By Theorem 3.1 of [FZ1], $\ddot{x}$ can be written as a Laurent polynomial in $x_{1}, \ldots, x_{n-1}, \dot{x}$. 
Since each cluster transformation is birational, $\dot{x}$ enters this polynomial with exponent 1 or -1 ; we write this as $\ddot{x}=\mathcal{L}_{0}+L_{1} \dot{x}^{ \pm 1}$ where $L_{0}$ and $L_{1}$ are Laurent polynomials in $x_{1}, \ldots, x_{n-1}$. Denote by $\ddot{x}^{\prime}$ the cluster variable in the cluster adjacent to $\ddot{\mathbf{x}}$ that replaces $\ddot{x}$, then

$$
\ddot{x}^{\prime}=\frac{M+N}{L_{0}+L_{1} \dot{x}^{ \pm 1}},
$$

where $M$ and $N$ are monomials in $x_{1}, \ldots, x_{n-1}$. Since $\ddot{x}^{\prime}$ is a Laurent polynomial in $x_{1}, \ldots, x_{n-1}, x$, we immediately get $L_{0}=0$.

We have to consider two cases: $\ddot{x}=L_{1} \dot{x}$ and $\ddot{x}=L_{1} / \dot{x}$. In the first case we write down $\dot{x}=L_{1}^{-1} \ddot{x}$ and apply once again Theorem 3.1 of [FZ1] to see that $L_{1}$ is a Laurent monomial $M_{+}$. In the second case we use (13) to get $\ddot{x}^{\prime}=M_{-} \dot{x}$ for some Laurent monomial $M_{-}$. It remains to prove that both $M_{+}$and $M_{-}$are identically equal to 1 . In the first case this would mean $\ddot{\mathbf{x}}$ and $\dot{\mathbf{x}}$ have $n$ common cluster variables, a contradiction. In the second case this would mean that $\dot{\mathbf{x}}$ is adjacent to $\ddot{\mathbf{x}}$ as required.

Assume that a variable $x_{i}$ enters $M_{+}$with a negative exponent $-k, k>0$. Let $x_{i}^{\prime}$ denote the cluster variable that replaces $x_{i}$ in the cluster adjacent to $\dot{\mathrm{x}}$ in the corresponding direction. Then $x_{i} x_{i}^{\prime}=M_{i}+N_{i}$, where $M_{i}$ and $N_{i}$ are monomials in $x_{1}, \ldots, x_{n-1}, \dot{x}$. Clearly, at least one of $M_{i}$ and $N_{i}$ is nontrivial, since otherwise the cluster algebra of rank 1 generated by $x_{i}$ would split off, in a contradiction to the assumption that $x_{i}$ enters $M_{+}$. Therefore,

$$
\ddot{x}=\frac{\dot{x}\left(x_{i}^{\prime}\right)^{k} M_{+}^{\prime}}{\left(M_{i}+N_{i}\right)^{k}},
$$

where $M_{+}^{\prime}$ is a Laurent monomial and the denominator is a nontrivial polynomial, which contradicts the Laurent property. To handle the case of a positive exponent $k>0$ we write $\dot{x}=M_{+}^{-1} \ddot{x}$ and proceed in a similar way using the variable $x_{i}^{\prime \prime}$ that replaces $x_{i}$ in the cluster adjacent to $\ddot{\mathrm{x}}$ in the corresponding direction. We thus obtained that $M_{+}$is a constant. It is an easy exercise to prove that the constant is equal to 1 . The case of $M_{-}$is handled similarly.

\section{Proof of Theorem 6}

Let $B_{\mathrm{pr}}$ be the $n \times 2 n$ matrix whose principal part equals $B$ and the remaining part is the $n \times n$ identity matrix. The corresponding algebra of geometric type $\mathcal{A}\left(B_{\mathrm{pr}}\right) \in$ $\mathfrak{A}(B)$ is called the algebra with principal coefficients and is denoted by $\mathcal{A}_{\mathrm{pr}}(B)$. Note that for this algebra initial coefficients $y_{1}, \ldots, y_{n}$ coincide with the stable variables $x_{n+1}, \ldots, x_{2 n}$.

The exchange graph of $\mathcal{A}^{\prime}$ covers the exchange graph of $\mathcal{A}$ if the equivalence of two seeds in $\mathcal{A}^{\prime}$ implies the equivalence of the corresponding two seeds in $\mathcal{A}$. It follows immediately from the definition that the exchange graph of any cluster algebra in $\mathfrak{A}(B)$ covers the exchange graph of the coefficient-free cluster algebra $\mathcal{A}_{\mathrm{cf}}(B)$. By Theorem 4.6 in [FZ4], the exchange graph of $\mathcal{A}_{\mathrm{pr}}(B)$ covers the exchange graph of any $\mathcal{A} \in \mathfrak{A}(B)$. Therefore, it suffices to prove that the exchange graphs for $\mathcal{A}_{\mathrm{pr}}(B)$ and $\mathcal{A}_{\mathrm{cf}}(B)$ coincide.

Define $X_{i ; \Sigma}\left(x_{1}, \ldots, x_{n} ; y_{1}, \ldots, y_{n}\right)$ as a rational function expressing the cluster variable $x_{i ; \Sigma}$ in $\mathcal{A}_{\mathrm{pr}}(B)$; further, define rational functions $G_{i ; \Sigma}$ by $G_{i ; \Sigma}\left(x_{1}, \ldots, x_{n}\right)=$ 
$X_{i ; \Sigma}\left(x_{1}, \ldots, x_{n} ; 1, \ldots, 1\right)$. By Theorem 3.7 in [FZ4], $G_{i ; \Sigma}$ express the variables $x_{i ; \Sigma}$ via the initial variables in the coefficient-free algebra $\mathcal{A}_{\mathrm{cf}}(B)$.

By Theorem 4, we have to prove the following implication: if

$$
G_{i ; \Sigma_{2}}=G_{\sigma(i) ; \Sigma_{1}}
$$

for some $\sigma \in S_{n}$, some seeds $\Sigma_{1}, \Sigma_{2}$ and any $i \in[1, n]$, then

$$
X_{i ; \Sigma_{2}}=X_{\sigma(i) ; \Sigma_{1}}
$$

for any $i \in[1, n]$. The proof is based on the following lemma.

Lemma 8. For any seed $\Sigma$ there exist Laurent monomials $M_{i ; \Sigma}$ such that

$$
\begin{aligned}
& X_{i ; \Sigma}\left(x_{1}, \ldots, x_{n} ; y_{1}, \ldots, y_{n}\right) \\
= & M_{i ; \Sigma}\left(z_{1}, \ldots, z_{n}\right) G_{i ; \Sigma}\left(x_{1} M_{1}\left(z_{1}, \ldots, z_{n}\right), \ldots, x_{n} M_{n}\left(z_{1}, \ldots, z_{n}\right)\right), \quad i \in[1, n],
\end{aligned}
$$

where $M_{i}=M_{i ; \Sigma_{0}}, \Sigma_{0}=(\mathbf{x}, \mathbf{y}, B)$ is the initial cluster and $z_{i}=y_{i}^{1 / \operatorname{det} B}$.

Proof. Consider toric actions on $\mathcal{A}_{\mathrm{pr}}(B)$ similar to those introduced in [GSV1]. Given an $n$-tuple of integer weights

$$
\mathbf{w}_{\Sigma}^{1}=\left(w_{1 ; \Sigma}^{1}, \ldots, w_{2 n ; \Sigma}^{1}\right), \ldots, \mathbf{w}_{\Sigma}^{n}=\left(w_{1 ; \Sigma}^{n}, \ldots, w_{2 n ; \Sigma}^{n}\right)
$$

for each seed $\Sigma$, define a local toric action by

$$
\left(x_{1 ; \Sigma}, \ldots, x_{2 n ; \Sigma}\right) \mapsto\left(t_{1}^{w_{1 ; \Sigma}^{1}} \ldots t_{n}^{w_{1 ; \Sigma}^{n}} x_{1 ; \Sigma}, \ldots, t_{1}^{w_{n ; \Sigma}^{1}} \ldots t_{n}^{w_{n ; \Sigma}^{n}} x_{n ; \Sigma}\right) .
$$

Two local toric actions at adjacent seeds are compatible if they commute with the transformation given by (5). If all local toric actions are compatible, they determine a global toric action on $\mathcal{A}(\widetilde{B})$ called the extension of each of the local actions.

A slight modification of Lemma 2.3 in [GSV1] guarantees that if

$$
\mathbf{w}^{1}=\left(w_{1}^{1}, \ldots, w_{2 n}^{1}\right), \ldots, \mathbf{w}^{n}=\left(w_{1}^{n}, \ldots, w_{2 n}^{n}\right)
$$

satisfy $\widetilde{B}\left(\mathbf{w}^{j}\right)^{T}=0$ for all $j \in[1, n]$ then the local toric action at $\Sigma_{0}$ defined by $\mathbf{w}^{1}, \ldots, \mathbf{w}^{n}$ can be extended to a global toric action.

Define the weights $\mathbf{w}^{i}$ as follows: the first $n$ entries of $\mathbf{w}^{i}$ constitute the $i$ th row of the matrix $B^{-1}$ multiplied by $\operatorname{det} B$, while its last $n$ entries constitute the $i$ th row of the $n \times n$-matrix $\operatorname{diag}(-\operatorname{det} B, \ldots,-\operatorname{det} B)$. Then the compatibility condition gives

$$
\begin{array}{r}
X_{i ; \Sigma}\left(x_{1} t_{1}^{w_{1}^{1}} \ldots t_{n}^{w_{1}^{n}}, \ldots, x_{n} t_{1}^{w_{n}^{1}} \ldots t_{n}^{w_{n}^{n}} ; y_{1} t_{1}^{-\operatorname{det} B}, \ldots, y_{n} t_{n}^{-\operatorname{det} B}\right) \\
=N_{i ; \Sigma}\left(t_{1}, \ldots, t_{n}\right) X_{i ; \Sigma}\left(x_{1}, \ldots, x_{n} ; y_{1}, \ldots, y_{n}\right)
\end{array}
$$

for some Laurent monomials $N_{i ; \Sigma}$. Define monomials $M_{i}$ by

$$
M_{i}\left(t_{1}, \ldots, t_{n}\right)=\prod_{j=1}^{n} t_{j}^{w_{j}^{i}} .
$$

Relation (15) follows from the above condition with $t_{i}=z_{i}$ and $M_{i ; \Sigma}=N_{i ; \Sigma}^{-1}$. 
From (14) and (15) we get

$$
X_{i ; \Sigma_{2}}=M_{i ; \Sigma_{2}} M_{\sigma(i) ; \Sigma_{1}}^{-1} X_{\sigma(i) ; \Sigma_{1}} .
$$

Let us prove that the monomial $M\left(y_{1}, \ldots, y_{n}\right)=M_{i ; \Sigma_{2}} M_{\sigma(i) ; \Sigma_{1}}^{-1}$ is, in fact, trivial, that is, equals 1.

Indeed, assume that there exists a variable, say, $y_{1}$, that enters $M$ with a negative exponent $-k, k>0$. Consider the $(n+1) \times 2 n$-matrix $\widehat{B}$ obtained from $\left(B_{\mathrm{pr}}\right)_{\Sigma_{1}}$ by adding the $(n+1)$ th row $\left(-d_{1} b_{1, n+1 ; \Sigma_{1}}, \ldots,-d_{n} b_{n, n+1 ; \Sigma_{1}}, 0, \ldots, 0\right)$, where $d_{1}, \ldots, d_{n}$ are the diagonal entries of $D$.

This matrix, together with the cluster $\widehat{\mathbf{x}}=\left(x_{1 ; \Sigma_{1}}, \ldots, x_{n ; \Sigma_{1}}, y_{1}\right)$ defines a cluster algebra $\mathcal{A}(\widehat{B})$ of rank $n+1$ with $m-1$ stable variables $y_{2}, \ldots, y_{m}$. Put $\widehat{\Sigma}_{1}=(\widehat{\mathbf{x}}, \widehat{B})$. The map $\hat{\cdot}$ is naturally extended to all seeds of $\mathcal{A}_{\mathrm{pr}}(B)$ so that if $\Sigma, \Sigma^{\prime}$ are adjacent in direction $k \in[1, n]$ then $\widehat{\Sigma}, \widehat{\Sigma}^{\prime}$ are adjacent in the same direction. Clearly, $\mathcal{A}_{\mathrm{pr}}(B)$ is the restriction of $\mathcal{A}(\widehat{B})$ to the first $n$ variables. Therefore, one can use only mutations in directions $1, \ldots, n$ in $\mathcal{A}(\widehat{B})$ to get

$$
x_{i ; \widehat{\Sigma}_{2}}=x_{n+1 ; \widehat{\Sigma}_{1}}^{-k} M^{\prime}\left(y_{2}, \ldots, y_{n}\right) x_{\sigma(i) ; \widehat{\Sigma}_{1}},
$$

where $x_{n+1 ; \Sigma_{1}}$ is naturally identified with $y_{1}$. The rest of the proof proceeds exactly as the proof of Theorem 5 .

\section{Acknowledgements}

The work on this project started during the visit of M. G. and M. S. to the Haifa Interdisciplinary Research Center for Advanced Computer Science in Fall 2006 and completed during the visit of A. V. to the Michigan State University in Spring 2007. The authors are grateful to these institutions for hospitality and stimulating atmosphere. The authors also thank A. Zelevinsky for attracting their attention to [BMRT].

\section{References}

[BFZ] A. Berenstein, S. Fomin, and A. Zelevinsky, Parametrizations of canonical bases and totally positive matrices. Adv. Math. 122 (1996), 49-149.

[BMRT] A. Buan, R. Marsh, I. Reiten, and G. Todorov, Clusters and seeds in acyclic cluster algebras. Proc. AMS. 135 (2007), no. 10, 3049-3060.

[FoG] V. Fock and A. Goncharov, Cluster ensembles, quantization and the dilogorathm, preprint, arxiv:math.AG/0311245.

[FST] S. Fomin, M. Shapiro, and D. Thurston, Cluster algebras and triangulated surfaces. Part I: Cluster complexes, To Appear, Acta Math..

[FZ1] S. Fomin and A. Zelevinsky, Cluster algebras.I. Foundations. J. Amer. Math. Soc. 15 (2002), $497-529$.

[FZ2] S. Fomin and A. Zelevinsky, Cluster algebras. II. Finite type classification. Invent. Math. 154 (2003), 63-121.

[FZ3] S. Fomin and A. Zelevinsky, Cluster algebras: notes for the CDM-03 conference, Current developments in mathematics, 2003, Int. Press, Somerville, MA, 2003, pp. 1-34.

[FZ4] S. Fomin and A. Zelevinsky, Cluster algebras. IV. Coefficients. Compos. Math. 143 (2007), $112-164$.

[GSV1] M. Gekhtman, M. Shapiro, and A. Vainshtein, Cluster algebras and Poisson geometry. Mosc. Math. J. 3 (2003), 899-934.

[GSV2] M. Gekhtman, M. Shapiro, and A. Vainshtein, Cluster algebras and Weil-Petersson forms. Duke Math. J. 127 (2005), 291-311. 
[Ze] A. Zelevinsky, Cluster algebras: origins, results and conjectures. Advances in algebra towards millennium problems, 2005, SAS Int. Publ., Delhi, pp. 85-105.

Department of Mathematics, University of Notre Dame, Notre Dame, Indiana 46556

Current address: Department of Mathematics, University of Notre Dame, Notre Dame, Indiana 46556

E-mail address: mgekhtma@nd.edu

Department of Mathematics, Michigan State University, East Lansing, Michigan 48823

E-mail address: mshapiro@math.msu.edu

Department of Mathematics AND Department of Computer Science, University of Haifa, Haifa, Mount Carmel 31905, Israel

E-mail address: alek@cs.haifa.ac.il 